\title{
Los estudios de trayectorias académicas y profesionales, contribuciones al estado del arte
}

Responsable de la investigación: Mtra. Irma Piña Jiménez., Colaboradoras en la investigación: EEAEC. Cristina Balán Gleaves**, EEAEC. Amelia Rodríguez Méndez***, EEAEC. Graciela Vázquez López****

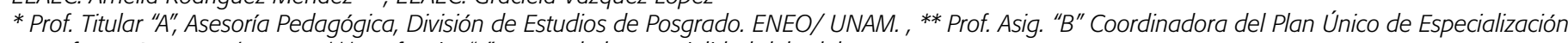
en Enfermería ENEO/ UNAM, *** Prof. Asig. "A" Tutora de la Especialidad del Adulto

en Estado Crítico ENEO/ UNAM, ****Prof. Asig. "A" Coordinador de la Especialización del Adulto en Estado Crítico, ENEO-UNAM

En este artículo se mencionan las características de una investigación que está en proceso de elaboración y que tiene el propósito de estudiar las trayectorias académicas y profesionales de tres generaciones de alumnos (2006, 2007 y 2008) que realizan los estudios de Especialización en Enfermería, en sus diversas orientaciones a través del Plan Único de Especialización en Enfermería (PUEE). Si bien se trata de una investigación preponderantemente de corte cuantitativo, se han reservado algunos aspectos para ser tratados en forma cualitativa. Se incluyen de manera sobresaliente los datos que aportó la primera fase de su elaboración, es decir la conformación de un estado del arte. Se adelantan los rubros que los instrumentos de investigación reúnen y cuya aplicación se ha realizado por ahora, para el caso de la cohorte 2006 y parcialmente en la del 2007.

PALABRAS CLAVE: Trayectorias académicas, eficiencia terminal, seguimiento de egresados.

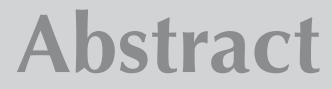

In this article are mentionated the characteristics of a research in process and which purpose is to study the academic and professional life of three generations (2006, 2007 and 2008). The students make or made their specialization in different branches of nursering through a syllabus called in Spanish Plan Único de Especialización en Enfermería (PUEE). Even though this research deals mainly with cuantitative aspects, some aspects have been reserved for their study based on a cualitative approach. The data from the first phase of researching are included, that is the antecedents. Also the characteristics of the research instruments applied to generation 2006 and 2007 are presented.

KEY WORDS: Academic history, graduates, students follow up.

\section{INTRODUCCIÓN}

Los estudios sobre las trayectorias académicas y profesionales, aportan sin duda una información valiosa para conocer cómo se presentan y comportan ciertas variables que están presentes en la población que se estudia y que favorecen u obstaculizan el buen desempeño del alumno en su estancia escolar. A mediano plazo el seguimiento de estas cohortes permiten realizar un seguimiento de sus egresados, permitiendo conocer a la institución que los forma, el impacto que ésta tiene en la vida profesional y personal de sus egresados, así como su repercusión en los servicios de salud a los que se incorporan.

En este sentido, se viene realizando una investigación de tres cohortes ${ }^{*}$ generacionales, bajo el título de Estudios longitudinales de trayectorias académicas y profesionales de los alumnos del Plan Único de Especialización en Enferme- 
ría. Estudio en el que se seguirán tres cohortes generacionales 2006, 2007 ( que actualmente cursa los estudios ) y la próxima generación 2008 que está por ingresar.

El objetivo general de esta investigación es realizar de manera sistemática el estudio de tres cohortes de alumnos que ingresan al PUEE en sus diversas especialidades* ${ }^{*}$, de manera que se puedan identificar las características de su ingreso, sus trayectorias académicas, su egreso y a mediano plazo su ubicación en el mercado de trabajo.

En este artículo se mencionan, algunas de las características que presenta esta investigación que está en proceso de desarrollo, destacando algunas reflexiones que son producto de la indagación y construcción de un estado del arte, así como las características que presentan los instrumentos de investigación que se han diseñado y que se vienen aplicando para tal fin.

\section{El Estado del Arte}

La revisión y el análisis de los estudios que se han realizado bajo esta temática en el nivel superior y en el posgrado, reflejan respecto al objeto en el que centran sus investigaciones, una preocupación constante por el hecho de determinar un elemento de predicción respecto a los resultados captados a través de los filtros por los que atraviesan los alumnos en el momento de su ingreso -es decir los exámenes de ingreso al nivel superior- y la posibilidad de permanecer y concluir exitosamente el programa educativo que realizan. En este caso pueden referirse los estudios de Benítez, Becerra y Soto' de la Universidad de San Luis Potosí o bien los de Chain Revuelta ${ }^{2}$ en la Universidad Veracruzana, en este último caso los estudios concluyen que el examen de selección no es suficiente para sostener una validez predictiva, en tanto confluyen otro conjunto de aspectos de los alumnos, durante el proceso formativo para explicar los resultados que obtienen.

Respecto a las metodologías de estudio empleadas, la ANUIES (Asociación Nacional de Universidades e Instituciones de Educación Superior) como instancia que trata de converger e integrar el trabajo conjunto de las Universidades y Escuelas de Educación Superior, conformó una metodología para la elaboración de estudios de egresados, $^{3}$ en la idea de aportar un instrumento de aplicación común que permita comparar los desempeños en las distintas instituciones de Educación Superior. El esquema que ofrece esta metodología además de incluir instrumentos para los alumnos, revisa la opinión de los empleadores, para analizar el impacto de los egresados en el mercado laboral, su movilidad social, espacial u ocupacional. Este último aspecto también es considerado en los estudios de Barranco y Santacruz ${ }^{4}$ y Oláiz y Ramírez ${ }^{5} e n$ la Universidad de Aguascalientes y en el Instituto de Salud Pública respectivamente.

Por su parte González Navarro ${ }^{6}$, propone el seguimiento de los alumnos desde una perspectiva de intervención psicopedagógica en las instituciones de Educación Superior, para lo cual adopta un modelo de sistemas de información. Este estudio tiene la bondad de recuperar información de un Departamento de Psicopedagogía para realizar una toma de decisiones más informada, en la idea de llevar un seguimiento constante de la evolución de los alumnos y orientarlos de manera más efectiva. Esto supone una intervención de apoyo al alumno durante su trayectoria escolar. Se trata de una experiencia realizada en la Universidad Autónoma de Baja California

Cabe también revisar en este sentido, el caso de Piña y Pontón, quienes destacan la relevancia de la vida Académica en la eficiencia terminal del Posgrado en Sociología de la UNAM. ${ }^{7}$

En función de las investigaciones revisadas, puede concluirse que los estudios sobre el seguimiento de los alumnos en su ingreso, permanencia y egreso, se perfilan en función de tres aspectos fundamentalmente: un primer aspecto que recupera los antecedentes académicos de los alumnos a través de exámenes de ingreso, en la idea de predecir que un buen nivel académico al inicio de los estudios, hipotéticamente permitirá predecir la posibilidad de una conclusión exitosa. Sin embargo este aspecto por si sólo no se sostiene por lo que se hace necesaria la presencia de un segundo aspecto, pues resultan tan importantes las características de ingreso como las de sostenimiento durante el trayecto de formación, que aseguren la presencia de condiciones idóneas para un buen rendimiento académico, es decir, no es suficiente contar con alumnos con buenos puntajes académicos en su ingreso, si paralelamente no se sostienen los apoyos necesarios durante el proceso educativo, y dentro de éstos apoyos aparecen tanto los de orden institucional que tienen que ver con los aspectos psicopedagógicos de las universidades y los entornos culturales y educativos que se ofrecen a los alumnos, como de los aspectos familiares, las condiciones socioeconómicas de vida, y el capital cultural que dan a los alumnos un respaldo y una oportunidad real de estudio.

Un tercer aspecto que se tiene en cuenta es el destino laboral de los egresados, de manera que las instituciones formadoras de Educación Superior, puedan llevar un 
seguimiento de los espacios laborales que ocupan sus egresados, con lo cual pueden identificarse numerosos aspectos, tales como principales sectores del mercado ocupacional que absorben a un mayor número de egresados, promedio de ingresos, proyecciones profesionales, puestos de trabajo, etc.

\section{Metodología e instrumentos de investigación}

Considerando el panorama anterior y regresando ahora a la investigación que nos ocupa, destacaremos que en esta investigación nos interesa identificar las características de ingreso que presentan los alumnos, dar seguimiento durante su proceso formativo en la especialidad e iniciar a mediano plazo su seguimiento como egresados, lo que consideramos nos permitirá tener una visión completa de todo el proceso y poder detectar aciertos y aspectos sobre los que es preciso poner atención o generar una mayor indagación.

Se trata en este caso de una investigación inicialmente de corte cuantitativo y como se mencionó anteriormente, nos hemos propuesto estudiar tres cohortes generacionales, es decir no se selecciona muestra alguna, se trabaja con toda la cohorte y se capta al alumno a su ingreso y se va siguiendo su trayectoria académica a lo largo de la especialidad. Para ello se diseñó un cuestionario que se aplica a los alumnos que han ingresado a las diversas especialidades de Enfermería, este instrumento contiene los siguientes rubros:

- Datos de identificación (Nombre, domicilio, edad, sexo, estado civil, etc.)

- Condiciones laborales al ingreso a la especialidad (lugar de trabajo, plaza que ocupa, antigüedad, si dispone de beca etc.)

- $\quad$ Antecedentes Académicos a nivel superior (Institución de procedencia, licenciatura realizada, año de titulación)

- Antecedentes académicos en el nivel de posgrado (si es el caso Institución y Programa cursado, año en que los concluyó y fecha de obtención del grado)

- Trayectoria Escolar (calificaciones obtenidas en cada actividad académica y promedio general))

- Eficiencia terminal (Opción de graduación elegida, fecha de la graduación).

Un segundo cuestionario con carácter más cualitativo de preguntas abiertas y totalmente anónimo, se elaboró y aplicó ya a la generación 2006, una vez que concluyó los estudios de la especialidad, con el propósito de reunir información sobre la percepción de estos alumnos a su egreso, respecto a los conocimientos fundamentales que obtuvieron durante la especialidad, así como sobre las posibilidades de aprendizaje que ofrecieron las sedes de prácticas y su nivel de satisfacción por los estudios concluidos.

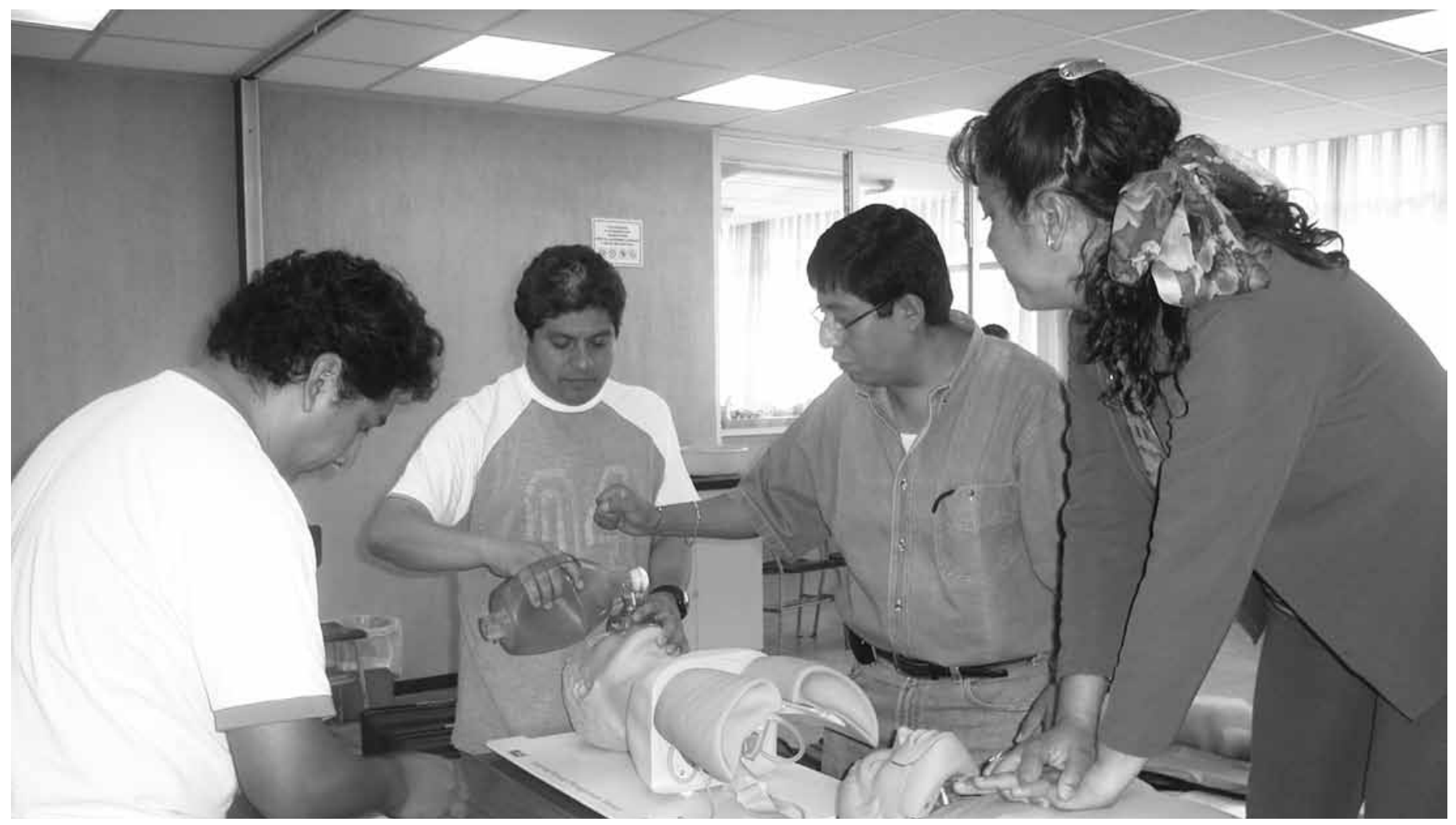


Un tercer instrumento será diseñado posteriormente, con el propósito de recuperar la información de los egresados, e iniciar de esta forma un estudio sistemático.

Por ahora ya se concluyó la elaboración de una base de datos, que permitirá analizar los datos que aportan los cuestionarios bajo el Programa Estadístico SPSS, de manera que podrá iniciarse ya la captura de la generación 2006 y 2007.

Paralelamente se inició el trabajo de captura del segundo cuestionario de preguntas abiertas. En futuras publicaciones, se dará a conocer un análisis de los resultados que arrojan los instrumentos de investigación.

\section{CONCLUSIONES}

La revisión y el análisis de documentos e investigaciones para conformar un estado del arte respecto a los estudios de ingreso, seguimiento de trayectorias académicas y egresados, muestran diversas metodologías si bien predominan las de corte cuantitativo. Se trata de estudios que constituyen un elemento por demás necesario, pues nos dan un mayor conocimiento sobre quiénes son nuestros alumnos, cómo transitan por sus estudios y qué hacen cuando los han concluido.

Esta información permite detectar tanto aciertos que consolida un programa educativo, como ubicar las tareas pendientes, pues si bien es necesario que ingresen alumnos con buenos niveles académicos, es también importante que un ambiente académico del programa educativo, sostenga su crecimiento y actúe sobre los problemas que obstaculizan su desempeño.

La investigación que estamos realizando con los alumnos de las especialidades de Enfermería del PUEE, aportará información valiosa y sistemática sobre estos aspectos y abrirá a nuevas incógnitas sobre las que será preciso profundizar, todo ello en razón de una búsqueda permanente de mejoramiento en la calidad educativa.

\section{NOTAS DE REFERENCIA}

${ }^{*}$ Cohorte es el conjunto de alumnos que ingresan en una carrera profesional o en un nivel de posgrado en un año determinado y cumple un trayecto escolar en un período normal en que prescribe el Plan de Estudios. La cohorte es la unidad fundamental del análisis estadístico, porque con base en ella se pueden agrupar y desagregar los datos referentes a los alumnos. Véase Barranco y Santacruz. **as especialidades que entran al estudio son: Enfermería en Salud Pública, Enfermería Cardiológica, Enfermería Perinatal, Enfermería del Adulto en Estado Crítico, Enfermería Infantil, Enfermería del Anciano, Enfermería en Rehabilitación, Enfermería en Salud Mental, Enfermería Neurológica y Enfermería en Cultura Física y Deporte.

\section{REFERENCIAS BIBLIOGRÁFICAS.}

1 Benítez Lima Ma. Gregoria, Gloria Eneida Becerra Quintero y Rosa Ma. Soto Luna. "Trayectorias escolares de los estudiantes de las generaciones 1998 y 1999 de la Facultad de Contaduría y Administración de la UASLP". En Revista de la Educación Superior. Vol. XXXIII (3), No. 131, Julio- Septiembre, 2004. p. 35

${ }^{2}$ Chain Revuelta, Ragueb. Examen de selección y probabilidad de éxito escolar en estudios superiores. Estudio en una universidad mexicana. Revista Electrónica de Investigación Educativa. 5 (1), 2003. 15 p. [En línea] disponible en http:// rediré,uabc.mx/vol5no1/contenido-chain.html Consultado el día 3 de octubre de 2005,

${ }^{3}$ ANUIES. Deserción, rezago y eficiencia terminal en las IES. Propuesta metodológica para su estudio. ANUIES, México, 2001. 244 p. (Serie Investigaciones)

${ }^{4}$ Barranco, R.S.M. y Santacruz, L.M. del C.(1995). Los egresados de la UAA. Trayectorias escolares y desempeño laboral. PIIES/ Universidad Autónoma de Aguascalientes.

${ }^{5}$ Olàiz Fernández Gustavo, Catalina Ramírez Cuadra, Oswaldo PALMA y Susana P. CERÓN (s/f). Sistema de Seguimiento de Egresados de los Programas de Posgrado del INSP/ ESPM Evaluación del desarrollo profesional y calidad de la educación. México, Instituto Nacional de Salud Pública. p. 5

${ }^{6}$ González Navarro, Félix Fernando. "Modelo de un sistema de información para el seguimiento psicopedagógico de alumnos en instituciones de Educación Superior". En Revista Iberoamericana de Educación. No. 35, Organización de los Estados Iberoamericanos para la Educación, la Ciencia y la Cultura, Madrid, 2005. p. 9

7 Piña Osorio, Juan Manuel Y Claudia Beatriz Pontón Ramos. "La eficiencia terminal y su relación con la vida Académica. El posgrado en Sociología y Ciencia Política de la UNAM", en Revista Mexicana de investigación Educativa. Enero- Junio, Vol 2, No. 3. 1997. pp. 85-102. 